\title{
Problèmes généraux de la dissipation d'émergie dans les ouvrages et circuits hydrauliques
}

\author{
General problems of energy dissipation \\ in hydraulic structures and systems
}

\author{
MM. Binder ${ }^{1}$, Duport $^{2}$, Meilland $^{3}$, Ramette ${ }^{4}$ et Valibouse ${ }^{2}$ \\ (1) Institut de Mécanique de Grenoble \\ (2) Alsthom-Atlantique, Neyrtec \\ (3) Sogreah \\ (4) Laboratoire National d'Hydraulique (EDF)
}

\section{Principales notations}

$E$ : énergie interne

$E_{i j}, e_{i j}:$ vitesse de déformation pure

$H: \frac{U_{i} U_{i}}{2}+P * / \rho=$ charge

$\ell$ : échelle de longueur des grosses structures turbulentes

$P:$ pression

$P^{*}: P+\rho g z$

$\mathscr{P}_{A}=-\rho \overline{u_{i} u_{j}} \overline{E_{i j}}:$ production de turbulence

$R_{M}: U \delta / \nu=$ nombre de Reynolds de l'écoulement moyen

$R_{T}=u^{\prime} \ell / \dot{\nu}=$ nombre de Reynolds de la turbulence $T$ : température

$T_{j}=\left(-\bar{P} *+2 \mu \overline{E_{i j}}-\rho \overline{u_{i} u_{j}}\right) \overline{U_{i}}$

$t_{j}=\left(-p+2 \mu e_{i j}-\rho u_{i} u_{j}\right) u_{i}$

$\mathcal{U}$ : vitesse caractéristique de l'écoulement moyen $U_{i},\left(\overline{U_{i}}\right), u_{i}=$ composantes de la vitesse instantanée, moyenne, fluctuante

$\bar{U}, \bar{V}$ : vitesses moyennes dans les directions $x$ et $y$

$u, v$ : fluctuations turbulentes de vitesse dans les directions $x$ et $y$

$\epsilon=2 E_{i j} E_{i j}:$ dissipation visqueuse

$\delta$ : échelle de longueur transversale de l'écoulement

$\eta$ : échelle de longueur des structures dissipatives

$\mu$ : viscosité dynamique

$\tau_{\ell}:$ temps de retoumement des structures turbulentes de dimension $\ell$

$\tau_{v}:$ temps de diffusion visqueuse

() : valeur moyenne lettre majuscule : valeur instantanée d'une grandeur lettre minuscule : fluctuation turbulente d'une grandeur ()$^{\prime}$ : écart type

()$_{M}$ : grandeur relative à l'écoulement moyen

()$_{T}$ : grandeur relative à l'écoulement turbulent.

Nécessité et imperatifs de la dissipation d'énergie hydraulique

Le sujet peut paraître provoquant aujourd'hui puisqu'on s'ingénie à récupérer toute énergie disponible.

Il existe cependant des cas où la dissipation d'énergie peut être admise en tant que telle parce qu'elle apparaît seulement une fraction du temps et avec des puissances très variables.

Malgré l'évolution des données économiques visant à des récupérations plus systématiques, il restera toujours un champ de préoccupation pour l'ingénieur dans la maîtrise de la dissipation d'énergie. Ceci mérite que nous y consacrions une certaine réflexion.

La fonction "dissipation d'énergie" peut ètre soit répartie le long de galerie ou de conduite sous forme de ce qu'on appelle généralement "perte de charge répartie" dans le conduit, soit concentrée dans un ouvrage spécialement conçu (dissipateur d'énergie hydraulique (DEH)).

Nous nous intéresserons essentiellement à ce dernier cas.

Le recours à des dissipateurs localisés résulte en général de la combinaison des circonstances suivantes:

(i) La fonction dissipation d'énergie fait suite à une fonction "réglage" (débit, niveau, pression), opérée au moyen de vannes, qui a pour effet de concentrer localement l'énergie à dissiper sous forme d'énergie cinétique. 
(ii) La dissipation d'énergie doit être organisée pour éviter les risques auxquels sont particulièrement exposés les ouvrages où s'effectue cette dégradation de l'énergie (vibrations, érosion par cavitation, érosion par les solides en suspension, affouillements...).

La construction d'un ouvrage, spécialement conçu pour concentrer la dissipation, est en général plus économique que le renforcement d'une grande longueur de canal ou de conduite.

Dans beaucoup d'installations hydrauliques, les ouvrages destinés à la fonction réglage (bloc vanne) sont nettement séparés des parties d'ouvrages jouant le rôle du DEH.

Par contre, dans les circuits en charge, il n'en est pas de même car la dissipation d'énergie est conditionnée par la conception de l'organe de réglage proprement dit. On est donc conduit à associer directement les fonctions du DEH à la fonction réglage.

Les exigences vis-à-vis du DEH concernent la stabilité globale de l'écoulement, la distribution de vitesse à l'aval (uniformité acceptable), le niveau et la taille de la turbulence affectant les ouvrages aval.

Par ailleurs, le DEH aura à satisfaire à deux contraintes: sa propre mécanique et la limitation de son coût.

\section{Processus de la dissipation d'énergie: la dissi- pation visqueuse}

La compréhension des problèmes que pose la concep. tion des D.E.H. passe évidemment par la compréhension des processus par lesquels l'énergie mécanique des écoulements se dissipe.

Nous examinerons d'abord la dissipation visqueuse dans les fluides incompressibles qui est évidemment le processus le plus largement présent dans les dissipateurs hydrauliques.

\section{Dissipation et perte de charge}

La dissipation apparait dans l'équation des forces vives, ou équation de l'énergie cinétique, qui s'obtient en effectuant le produit scalaire de l'équation de la dynamique par la vitesse. Pour un fluide incompressible, homogène, newtonien, cette équation peut s'écrire sous la forme :

$$
\rho \frac{d H}{d t}=\rho \frac{d}{d t}\left(\frac{U_{i} U_{i}}{2}+\frac{P^{*}}{\rho}\right)=\frac{\partial P}{\partial t}+\frac{\partial}{\partial x_{j}}\left(2 \mu U_{i} E_{i j}\right)-\epsilon
$$

$H$ étant la charge, $E_{i j}$ la vitesse de déformation pure et $\epsilon$ la dissipation visqueuse

$\epsilon=2 \mu E_{i j} E_{i j}=\mu \frac{\partial U_{i}}{\partial x_{j}} \frac{\partial U_{i}}{\partial x_{j}}+\mu \frac{\partial^{2}}{\partial x_{i} \partial x_{j}}\left(U_{i} U_{j}\right)$

Puisque la dissipation $\epsilon$ est une somme de carrés d'après la première expression, elle est positive et se traduit toujours par une perte de charge.

La conservation de l'énergie ( $1^{\text {er }}$ principe) peut d'autre part s'écrire sous la forme :

$$
\rho \frac{d E}{d t}=\rho C \frac{d T}{d t}=\frac{\partial}{\partial x_{j}} k \frac{\partial T}{\partial x_{j}}+\epsilon
$$

La dissipation se traduit toujours par un accroissement de l'énergie interne $E$ du fluide, c'est-à-dire de sa température. Elle correspond donc à une dégradation d'énergie mécanique en chaleur.

\section{Taux de dissipation et production de turbulence}

Dans les DEH, l'essentiel de la dissipation est effectué par la turbulence. Pour mettre en lumière des mécanismes de ce processus, il convient de séparer les diverses grandeurs en valeur moyenne et fluctuation turbulente et d'effectuer la moyenne statistique des équations.

En comptabilisant séparément les contributions du mouvement moyen et de la turbulence aux forces vives, on peut écrire après quelques manipulations algébriques $(1,2)$ les équations des bilans de l'énergie cinétique du mouvement moyen et de la turbulence :

$$
\begin{gathered}
\rho \frac{d}{d t}\left(\overline{\frac{U_{i} U_{i}}{2}}\right)=\frac{\partial}{\partial x_{j}} T_{j}+\rho \overline{u_{i} u_{j}} \overline{E_{i j}}-\epsilon_{M} \\
\rho \frac{d}{d t} \frac{\overline{u_{i} u_{i}}}{2}=\frac{\partial}{\partial x_{j}} t_{j}-\rho \overline{u_{i} u_{j}} \overline{E_{i j}}-\epsilon_{T}
\end{gathered}
$$

où $T_{j}$ et $t_{j}$ désignent l'ensemble des termes dus aux travaux des contraintes (pression, contraintes visqueuses et contraintes turbulentes ou de Reynolds $-\rho \overline{u_{i} u_{j}}$ ). $\epsilon_{M}$ et $\epsilon_{T}$ sont les dissipations produites par le mouvement moyen et par la turbulence. Nous allons voir que $\epsilon_{T} \gg \epsilon_{M}$.

Dans chacune des équations ci-dessus apparaît le terme $\mathscr{T}_{R}=-\rho \overline{u_{i} u_{j}} E_{i j}$ mais avec un signe différent. En général $\mathscr{P}_{n}>0$, et ce terme tend donc à maintenir l'énergie cinétique de la turbulence contre l'action de la dissipation $\epsilon_{T}$, d'ou son appellation: "production et turbulence", et il fait décroître l'énergie cinétique du mouvement moyen. Il correspond donc à un mécanisme de transfert d'énergie du mouvement moyen à la turbulence.

L'importance relative de $\mathscr{P}_{\text {et }}$ de $\epsilon_{M}$ dans (3) peut être clairement évaluée dans les écoulements cisaillés minces qui recouvrent de nombreux écoulements pratiques, notamment en hydraulique (écoulements en conduite ou dans les canaux, couches limites, jets, sillages). Dans ces écoulements, les gradients transversaux sont beaucoup plus raides que les gradients longitudinaux:

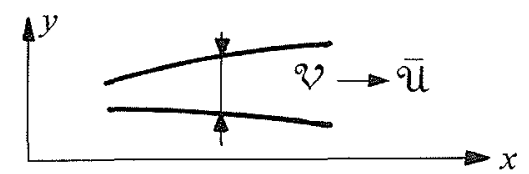

$\frac{\partial}{\partial y} \gg \frac{\partial}{\partial x}$ ce qui implique que $U \gg V$ et que le terme dominant de $\overline{E_{i j}}$ est $\frac{\partial \bar{U}}{\partial y}$.

Si $\mathcal{U}$ caractérise les vitesses moyennes et si $\vartheta$ est la 
largeur de l'écoulement on a :

$$
\overline{E_{i j}} \sim \frac{\partial \bar{U}}{\partial y} \sim \frac{\mathcal{U}}{\vartheta} \quad \text { et } \quad \mathscr{P} \simeq-\rho \overline{u v} \frac{\partial u}{\partial y} \sim-\rho \overline{u v} \frac{\mathcal{u}}{\vartheta}
$$

L'expérience montre par ailleurs que les fluctuations $u$ et $v$ sont bien correlées, à savoir que $\overline{u v}=C u^{\prime 2}$ avec $C=0$ (1) (11) où $u^{\prime}=\sqrt{u_{i}} u_{i} / 2$ est l'échelle de vitesse caractéristique de l'énergie cinétique turbulente. Une valeur typique est $C=0,25$, mais ce coefficient peut varier à travers un écoulement et il est en particulier nul dans un plan de symétrie. On a par suite :

$$
\frac{\epsilon_{M}}{\mathscr{P}} \sim \frac{\mu(\mathcal{U} / \mathcal{V})^{2}}{\rho u^{\prime 2} \mathcal{U} / \mathcal{V}}=\left(\frac{\mathscr{V}}{u^{\prime}}\right)^{2} \frac{\nu}{\mathcal{U} \mathcal{V}}
$$

comme $\frac{u^{\prime}}{u} \sim 1 / 10$ et que le nombre de Reynolds

$$
R_{M}=\frac{\text { U? }}{\nu} \gg 1
$$

il en résulte que :

$$
\epsilon_{M} / \mathscr{\rho} \ll 1
$$

Dans le bilan de l'énergie cinétique moyenne (3), la dissipation $\epsilon_{M}$ produite par l'écoulement moyen luimême est insignifiante par rapport à la production d'énergie cinétique turbulente.

Le terme $\frac{\partial}{\partial x_{i}} t_{j}$ dans le bilan de l'énergie cinétique turbulente (4) correspond simplement à une diffusion d'une région vers une autre et il ne joue en général aucun rôle dans un bilan global. Comme l'énergie cinétique de la turbulence est bornée, il faut que :

$$
\epsilon_{T} \sim \mathscr{P}>\rho \frac{d}{d t} \frac{\overline{u_{i} u_{i}}}{2}
$$

Dans un écoulement établi, par exemple :

$$
\rho \frac{d}{d t} \frac{\overline{u_{i} u_{i}}}{2}=0
$$

le taux de dissipation est donc fixé par la production. II est clair que : $\epsilon_{T} \gg \epsilon_{M}$ toute la dissipation est pratiquement effectuée par la turbulence. D'après (5), (6) et (8), on peut évaluer $\epsilon_{T}$ par:

$$
\epsilon_{T} \sim \rho u^{\prime 2} \Psi / \vartheta
$$

Appelons $\mathscr{E}_{\ell}=\ell / u^{\prime}$ le temps de retournement des gros tourbillons de dimension $\ell$. Les mesures montrent que :

$$
\ell / u^{\prime} \sim \vartheta / \mathcal{U}
$$

Cette égalité approximative entre le temps de retour. nement et le temps caractéristique de l'écoulement moyen $\vartheta / U$ qui peut s'interpréter physiquement comme le temps nécessaire pour allonger appréciablement un élément fluide $\left(\overline{E_{i j}} \sim \mathcal{U} / \mathcal{V}^{\gamma}\right)$ peut se comprendre puisque la production de turbulence s'effectue précisément par l'allongement des filets tourbillon. Il résulte finalement de $(8),(9)$ et $(10)$ :

$$
\epsilon_{T} \sim \mathscr{P} \sim \frac{\rho u^{\prime 3}}{\ell}
$$

La signification physique de $\epsilon_{T}$ est une perte d'énergie cinétique par unité de temps :

$$
\epsilon_{T} \sim \frac{\Delta\left(\rho v^{2}\right)}{\Delta t}
$$

On voit que si on prend $\Delta\left(\rho v^{2}\right) \sim \rho u^{\prime 2}$, le taux de dissipation ci-dessus impose que :

$$
\begin{aligned}
& \Delta t \sim \mathscr{\mho}_{\ell} \\
& \epsilon_{T} \sim \frac{\rho u^{\prime 2}}{\mathscr{C}_{\ell}}
\end{aligned}
$$

Les gros tourbillons perdent donc une fraction importante de leur énergie cinétique pendant un temps de retoumement. Cette conclusion est directement confirmée par les expériences de turbulence derrière une grille où le seul mécanisme actif est la dissipation, la production étant nulle puisqu'il n'y a pas de gradient de vitesse moyenne. Dans cette turbulence livrée à ellemême, la dissipation peut être déterminée par la décroissance de l'énergie cinétique :

$$
\rho \frac{d u^{\prime 2}}{d t}=-\epsilon_{T}
$$

\section{Les échelles dissipatives}

Le résultat ci-dessus (11), nous renseigne sur le taux de dissipation, mais ne révèle rien sur son mécanisme. Comme la dissipation est un phénomène visqueux, calculons le temps $\tau_{v}$ qu'il faudrait pour que le frottement visqueux à lui seul fasse perdre à un gros tourbillon son énergie cinétique. Si on pouvait instantanément geler le fluide autour du tourbillon de dimension $\ell$, le temps $t_{v}$ est celui qui est nécessaire pour que le frottement visqueux diffuse de la périphérie au centre :

$$
\tau_{v} \sim \ell^{2} / \nu
$$

et rapporté au temps de retournement initial :

$$
\frac{\tau_{\nu}}{\tau_{\ell}} \sim \frac{\ell^{2} / \nu}{\ell / u^{\prime}}=\frac{\ell u^{\prime}}{\nu}=R_{T} \gg 1
$$

$R_{T}$ est le nombre de Reynolds des grosses structures de la turbulence $\left(R_{T} \sim 1 / 10 R_{M}\right)$. Ce nombre de Reynolds est donc le nombre de tours nécessaires pour freiner un gros tourbillon par frottement visqueux.

Or, le taux de dissipation (12) montre que la perte d'énergie de ces tourbillons s'effectue pendant un temps de retoumement, d'où la conclusion que ces tourbillons perdent leur énergie par un autre mécanisme. Dans les écoulements considérés (incompressibles et homogènes), il n'y $\mathrm{a}$, en dehors des mécanismes visqueux, que les mécanismes "inertiels" (ou de fluide parfait) qui puissent agir. Ils résultent des intéractions des divers tourbillons entre eux qui pro- 
duisent des allongements et des rétrécissements des filets tourbillon, qui se traduisent par un gain d'énergie cinétique des structures de taille inférieure.

Ces structures à leur tour fabriquent des tourbillons plus petits au détriment de leur propre énergie cinétique, etc. C'est la cascade de l'énergie des grosses vers les petites structures. Cette cascade se prolonge jusqu'à ce que les structures soient suffisamment petites pour dissiper le taux imposé $\epsilon_{T} \sim u^{\prime 3} / \ell$.

Si l'on désigne par $\eta$ et $\nu$ leurs échelles de longueur et de vitesse, appelées échelles de Kolmogorov, on a d'après la définition même de la dissipation (2) :

$$
\epsilon_{T}=\mu \frac{v^{2}}{\eta^{2}}
$$

De plus, ces petites structures dissipatives doivent être telles qu'elles perdent leur énergie cinétique par effet visqueux pendant un temps de retoumement $\eta / \nu$, sinon elles pourraient engendrer des tourbillons encore plus petits. Elles doivent donc avoit un nombre de Reynolds égal à un $\nu \eta / \nu=1 \quad$ (14).

Compte-tenu de $(11),(13)$ et (14), on trouve alors:

$$
\begin{array}{lll}
\eta=\left(\frac{\rho \nu^{3}}{\epsilon_{T}}\right)^{1 / 4} & v=\left(\frac{\epsilon_{T} \nu}{\rho}\right)^{1 / 4} \\
\frac{\eta}{\ell} \sim R_{T}^{-3 / 4} & \frac{v}{u^{\prime}} \sim R_{T}^{-1 / 4}
\end{array}
$$

En résumé, les mécanismes de la dissipation turbulente sont les suivants :

- la perte de charge de l'écoulement moyen est faite par la production de turbulence à un taux $\rho u^{\prime 3} / \ell$; - qui entretient les grosses structures turbulentes; - dont les interactions font cascader l'énergie cinétique vers les petites structures ;

- jusqu'à ce que leurs dimensions soient suffisamment petites pour dissiper le taux d'énergie $\rho u^{\prime 3} / \ell$ imposé par la production.

\section{Conséquences pratiques}

Pour le dimensionnement des ouvrages dissipateurs, les résultats essentiels peuvent se résumer par les relations approximatives suivantes :

$$
-\rho \frac{d \bar{H}}{d t}=\mathscr{S}_{A} \simeq \epsilon_{T} \sim u^{2} \mathcal{U} / \vartheta \sim u^{\prime 3} / \ell
$$

Pour obtenir une perte de charge rapide, il faut intensifier au maximum le taux de production turbulente par unité de volume. Ceci peut être accompli en accroissant les gradients de vitesse moyenne, notamment les gradients transversaux $\mathcal{U} / \mathcal{Q}$ en jouant si possible sur le module de vitesse et surtout sur l'échelle transversale de longueur, d'où l'intérêt, bien connu de l'ingénieur, de fractionner la veine en jets ou sillages multiples, ce qu'on rencontre dans des vannes à trous comme le Monovar développé par Neyrtec.

Le dernier facteur par lequel on peut penser intervenir est 1 'intensité turbulente $u^{\prime 2}$, mais cette dernière est liée à la vitesse moyenne $\mathcal{U}$ et est pratiquement fixée par le type de l'écoulement élémentaire produit. Par exemple un jet est nettement plus dissi- pateur qu'une couche limite, puisque les taux de turbulence $u^{\prime} / \mathcal{U}$ sont respectivement de 25 et $10 \%$.

Enfin, on notera encore que la longueur $L$ nécessaire à l'amortissement de la turbulence excédentaire créé par un organe dissipateur sera proportionnelle à :

$$
V \tau_{\ell_{\max }} \simeq \frac{V}{u^{\prime}} \ell_{\max }
$$

où $V$ est la vitesse moyenne de la veine, $\tau_{\ell_{\max }}$ le temps caractéristique des plus grosses structures turbulentes produites de dimension $\ell_{\max }$. S'il faut renforcer un ouvrage après un dissipateur, à cause des effets néfastes produits par les fortes fluctuations, la longueur à prendre en compte sera $L$. La constante numérique est fixée empiriquement en fonction de la perte de charge maximum à dissiper et du type d'ouvrage.

\section{Processus de dissipation par entraînement d'air}

Il existe un assez grand nombre de circonstances où la dissipation d'énergie s'accompagne d'un entraînement d'air dans l'écoulement et de la formation de zones où l'écoulement est diphasique du type à phase gazeuse dispersée dans l'eau.

L'entraînement d'air peut être spontané (ressaut, écoulement rapide à surface libre, jet libre plongeant dans un bassin...) ou résulter de dispositions intentionnellement adoptées (reniflard derrière une vanne, aération "par en dessous" d'une nappe à grande vitesse sur un coursier, aération d'un jet noyé etc.).

On attribue à juste titre à ces zones d'écoulement diphasique des effets bénéfiques: réduction ou élimination du risque d'érosion de cavitation, réduction de l'amplitude des fluctuations de pression.

On a constaté également que la dissipation d'énergie est notablement plus intense lorsque le processus de dissipation s'accompagne d'un fort émulsionnement. C'est ainsi qu'avec des vannes à disques dénoyés, où l'impact du jet dans le bassin provoque un fort entraînement d'air, on dissipe dans un volume donné, des puissances au moins trois fois plus élevées que dans la disposition à vanne noyée.

A notre connaissance, plusieurs processus peuvent être invoqués pour expliquer les effets globaux constatés :

\section{Le glissement de phase}

qui est une source de dissipation proprement hydrodynamique : chaque bulle est animée, par rapport au liquide qui l'entoure, d'un mouvement relatif (glissement) en direction des zones à pression plus basse.

Cette dissipation est cependant assez limitée lorsque le seul gradient est le gradient hydrostatique.

\section{Le blocage diphasique}

La dissipation par glissement de phase peut, par contre, etre beaucoup plus intense s'il existe des gradients dynamiques de pression positifs très élevés (pression croissante dans le sens de l'écoulement général); 
or, c'est précisément ce qui se produit lorsqu'on est en présence de blocage diphasique. Nous disposons donc là d'un second processus dissipatif dont l'effet ne s'explique sans doute pas seulement par l'intensification locale du glissement.

Les vitesses d'écoulement au sein des DEH sont en général notablement supérieures à la célérité du son dans le fluide diphasique, ce qui donne lieu à des chocs (en général assez diffus) et à des zones de blocage où le glissement de phase est considérablement amplifié. $\mathrm{Au}$ cours d'une expérience récente, en laboratoire, des taux de dissipation de plusieurs dizaines de $\mathrm{kW} / \mathrm{m}^{3}$ ont été observés.

\section{L'amortissement thermique}

Dans les processus décrits ci-dessus, on suppose que la pression dans la bulle suit instantanément la pression locale ambiante et que la température interne de la bulle est homogène et égale à la température du liquide.

Or, au regard de variations suffisamment rapides de pression (turbulence, "choc diphasique"), ces hypothèses ne sont pas vérifiées.

Pour une large bande de fréquences, l'évolution de la température dans la bulle est polytropique, ce qui entraîne un effet d'hystérésis et un transfert d'énergie calorifique du gaz vers le liquide.

Pour des fréquences de fluctuations de pression proches de la résonance des bulles, des dissipations de plusieurs dizaines de $\mathrm{kW} / \mathrm{m}^{3}$ peuvent ètre facilement expliquées.

\section{Aspects communs des problèmes technologiques et divers types de solutions Problèmes de tenue des DEH}

\section{Cavitation}

Le risque d'érosion de cavitation est toujours présent en raison des circonstances suivantes:

- la dissipation d'énergie hydraulique passe en général par la transformation préalable de cette énergie en énergie cinétique ;

- la création de la turbulence nécessaire pour dissiper cette énergie entraîne des fluctuations de pression importantes.

Le second effet est illustré par la cavitation de mélange qui prend naissance autour des jets noyés ou dans les confluences à fort cisaillement alors que la pression moyenne est notablement supérieure à la tension de vapeur.

On résout le problème du risque d'érosion de cavitation :

- en fractionnant la perte de charge en plusieurs étages ; - en revêtant les parois du DEH par des blindages métalliques ;

- en injectant de l'air (selon un procédé breveté par Neyrtec). L'inhibition de l'érosion peut être obtenue par l'injection contrôlée de très faibles quantités d'air.

\section{Vibrations}

Les risques de vibrations auxquels sont exposées les parois et les structures des DEH proviennent des processus mêmes de la dissipation d'énergie :

- génération d'écoulement à grande vitesse,

- création et entretien d'un niveau élevé de turbulence.

Les fluctuations de pression liées aux taux élevés de turbulence peuvent être à l'origine de vibrations de première espèce (au sens du Professeur Fortier).

L'existence de zones d'écoulement à grande vitesse peut être à l'origine de phénomènes de couplage où l'énergie nécessaire à l'entretien de la vibration est prélevée sur l'énergie "continue" de l'écoulement rapide. La prévision de ces vibrations de deuxième espèce ne peut être faite par les analyses séparées des sollicitations hydrauliques et de la réponse des structures.

Parmi les solutions techniques permettant d'éloigner le risque de vibrations, nous retiendrons principalement :

- la fragmentation transversale des écoulements : Ce fractionnement est favorable à l'intensification du taux de production turbulente par unité de volume. Il est également à l'origine de la réduction de taille des grosses structures turbulentes, potentiellement les plus actives dans la génération de vibrations;

- la ventilation des écoulements : Elle peut agir favorablement par l'effet déjà signalé de réduction de l'intensité des fluctuations de pression liées aux grosses structures turbulentes; elle peut aussi agir par la suppression d'instabilités globales d'écoulement.

\section{Affouillements}

Lorsque les parois d'un ouvrage dissipateur d'énergie ne sont pas parfaitement étanches (roche en place, revêtement maçonné ou même en béton si celui-ci est fissuré), l'existence d'écoulements à grande vitesse peut être à l'origine d'affouillement mettant en cause la pérennité de l'ouvrage.

L'affouillement est déclenché par la pénćtration locale de pressions elevées (ordre de grandeur de la pression d'arrêt) sous le revêtement.

Les dispositions techniques déjà mentionnées : fractionnement transversal des écoulements, étagement de la dissipation, ventilation des écoulements, protection par blindages métalliques, contribuent, en plus de leurs rôles déjà décrits, à la prévention du risque d'affouillement.

Citons également deux solutions propres aux DEH à jet libre :

- protection contre l'impact direct du jet par un bassin de dissipation placé dans la zone de retombée du jet; - dispersion du jet soit par l'emploi de certains types de vannes (vannes à jet creux par exemple), soit par ventilation du jet. Cette dispersion du jet ne participe que fort peu à la dissipation d'énergie. C'est un processus de fragmentation des écoulements ayant pour effet de dissiper l'énergie sur des distances beaucoup plus courtes, ce qui réduit l'épaisseur du "matelas d'eau" nécessaire. 


\section{Application aux ouvrages dissipateurs d'énergie}

Les appareils de dissipation d'énergie dans les circuits font l'objet de plusieurs communications de cette session du Comité Technique. Les ouvrages dissipateurs par contre, ne sont qu'indirectement abordés. Nous proposons donc de dresser un tableau succinct des types de dispositions que l'on rencontre fréquemment dans ce genre d'ouvrages afin d'illustrer ce qu'il y a de commun à l'ensemble des DEH en ce qui concerne les phénomènes mis en jeu (processus de dissipation, phénomènes mettant en cause la tenue des ouvrages : cavitation, abrasion, affouillement...) et les solutions hydrauliques mises en œuvre.

\section{Conclusions}

Il est intéressant de constater que certaines dispositions fréquemment rencontrées : fragmentation des écoulements, ventilation des écoulements et des bassins de dissipation, bien qu'issus d'une longue expérience qui doit plus à l'art de l'ingénieur qu'à la déduction scientifique, s'expliquent en fait fort bien par l'analyse des phénomènes en cause (dissipation visqueuse, génération de la turbulence, dissipation diphasique). Il y a là un sujet de réflexion qui nous paraît à méditer car le rapprochement entre les technologies et les faits scientifiques pourrait bien, dans ces domaines comme dans beaucoup d'autres, être potentiellement générateur de nouveaux progrès à venir.

\section{Références}

TENNEKES H. et LUMLEY J.L. - A first course in turbulence, MIT Press, 1972.

FAVRE A., KOVASZNAY L.S.G., DUMAS R. et al. - La turbulence en Mécanique des Fluides, Gauthier Villars, 1976.

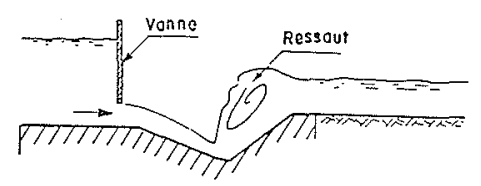

Figure 1. - Barrage au fil de 1'eau comportant, comme ouvrage dissipateur, un bassin à ressaut.

La turbulence est engendrée par le ressaut ; en raison de l'entraînement d'air par ce dernier, il peut également exister un processus diphasique de dissipation.
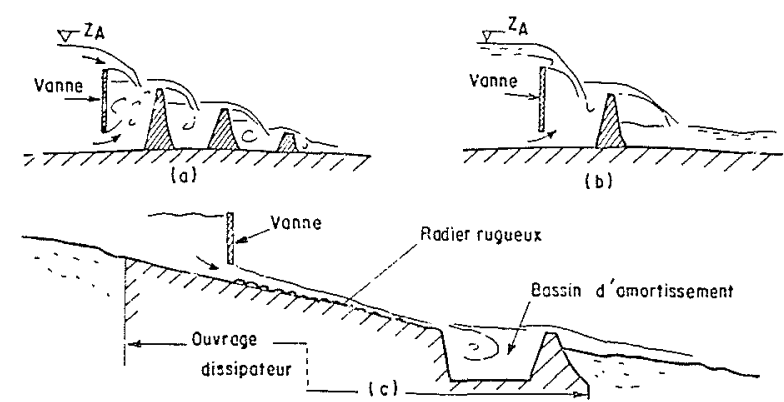

Figure 2. - Les trois dispositions représentées illustrent le recours au fractionnement longitudinal de la dissipation.

- Processus de génération de la turbulence par jets noyés et par radier rugueux (C).

- Le processus de dissipation diphasique existe à coup sûr aussi (entraînement d'air par jet plongeant), et aération spontanée de l'écoulement rapide (a) - (b) - (c).
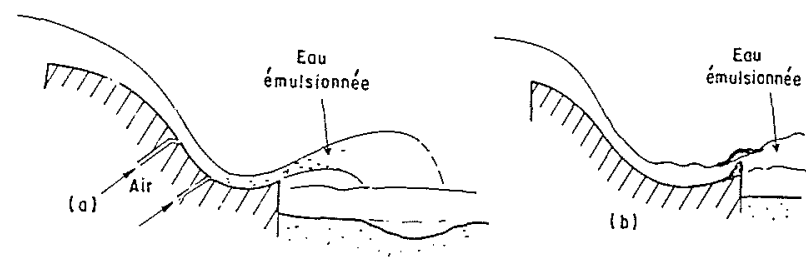

Figure 3. - Evacuateur à "saut de ski" suivi de bassins de dissipation.

Admission d'air sur le radier du coursier de la disposition (protection contre l'érosion par cavitation, dispersion du jet, dissipation d'énergie par effet diphasique). 


\title{
Discussion
}

\author{
Président : M. P.BERGERON
}

M. le Président. - Vous venez d'entendre cet exposé très complet de la question, qui passe de la théorie aux aspects communs des problèmes technologiques.

Cette communication est très intéressante en début de Session. Les autres communications des trois demi-journées, qui vont suivre, porteront surtout sur les réalisations industrielles.

J'ouvre la discussion sur le sujet.

M. LEFOULON. - Deux remarques à titre d'exemples de dissipateur d'énergie étudiés sur modèle réduit à Chatou il y a trente ans:

1) Pour supprimer les vibrations de la lame d'eau déversante d'un barrage du Massif Central (Le Gournoir) on a simplement placé sur la crête des becs de corbeau. Ceux-ci divisaient la lame d'eau en plusieurs tranches qui étaient ainsi aérées ce qui supprimait la vibration.

2) Pour dissiper l'énergie des déchargeurs des centrales du Rhin à partir de Fessenheim, on a disposé des barreaux dans les coursiers à l'aval des vannes-secteurs de décharge. Leur nombre et leur emplacement ont été étudiés sur modèle réduit. La turbulence obtenue réduisait la vitesse d'écoulement à la sortie du coursier à une vitesse qui évitait toute érosion dans le canal de fuite. Je rappelle que le déchargeur doit restituer le débit d'un groupe en cas d'arrêt inopiné de celui-ci, pour éviter tou te intumescence préjudiciable à la navigation dans le canal d'amenée.

$M$. le Président. - Les spécialistes de la turbulence ici présents peuvent-i is me donner l'explication du phénomène suivant constaté expérimentalement?

Pour un diaphragme de mesure de débit, la mesure n'est absolument pas modifiée par l'apparition de la cavitation autour du jet contracté, et ceci pratiquement jusqu'à la cavitation globale qui modifie le comportement de l'écoulement à l'aval du jet. De plus, on peut constater que la perte et la récupération de pression à la Borda restent inchangées pendant toute l'évolution du phénomène.

Il faut en conclure que la perte reste constante et que l'énergie se dissipe de la même manière, dans un volume qui lui-même reste constant, mais dont l'état passe du liquide homogène à un mélange diphasique de plus en plus accentué.

M. DUPORT. - La remarque du Président BERGERON est très intéressante; elle me donne l'occasion d'apporter une précision.

Tout à l'heure, j'ai parlé đ'écoulement diphasique à phase gazeuse dispersée dans le liquide; j'aurais dû ajouter : sans changement de phase important. En effet, toutes les considérations auxquelles $j$ 'ai fait allusion - car je ne les ai pas vraiment développées - valent pour ce cas : écoulement à phase gazeuse dispersée sans changement de phase.

Pour l'écoulement cavitant à cavitation développée dont a parlé M. BERGERON, je vais me permettre de dessiner un diaphragme tout simple, avec des mesures de pression à l'aval immédiat, dans la zone contractée. A l'endroit qui est normalisé pour la mesure, il va se produire - même pour des $\sigma$ relativement élevés, c'est-à-dire alors même que la pression moyenne est notablement supérieure à la pression de vapeur, donc bien avant le blocage par cavitation globale - de la cavitation de mélange comme celle dont j'ai parlé tout à l'heure. Cet écoulement est localement diphasique par suite de la présence de bulles de vapeur, ou, plus exactement, de filets de tourbillon cavitants. Ces filets de tourbillon vont disparaître dès que le tourbillon lui-même aura été amorti par viscosité, sans doute un peu modifiée par la présence de la cavitation. En fait, il n'y aura pas transport d'écoulement diphasique vers l'aval tout au plus, en présence d'eau naturelle, va-t-il y avoir une certaine extraction de gaz dissous. Très souvent on constate, à l'aval, si on observe avec soin, que l'écoulement contient un peu d'émulsion, mais il s'agit alors d'une émulsion à taux de vides relativement bas et pour laquelle l'influence du diphasique est tout à fait différente de celle à laquelle j'ai fait allusion

Par conséquent, il n'y a pas réellement modification du processus de dissipation, et il n'est pas tellement étonnant qu'on ait constaté, comme le disait M. BERGERON, des pertes de charges qui sont les mêmes dans l'écoulement subcavitant que celles que donne la formule de Borda. En fait, c'est seulement au moment où apparaît la cavitation globale que l'on a des conditions tout à fait différentes. J'en parle peu parce qu'une des communications de cette matinée ou de cet après-midi traite d'appareils qui suivent ce principe.

M. le Président. - Vous avez défini le coefficient de cavitation en prenant en considération la pression amont et la perte de charge comme le font les constructeurs des organes de réglage et de dissipation d'énergie, ce qui est normal quand on veut étudier le fonctionnement d'un appareil particulier. Mais pour évaluer l'efficacité relative, par exemple, de différents types de diaphragmes, nous avions préféré adopter

$$
\sigma=\frac{\left(P_{j} / \omega\right)-t_{v}}{V_{j}^{2} / 2 g}
$$

$P_{j}$ étant la pression au droit de la veine ou des veines contractées, et $V_{j}$ la vitesse dans cette veine évaluée en appliquant Bernoulli. Quelqu'un d'autre souhaite-t-il prendre la parole?

M. REMENIERAS. - A titre d'illustration du processus de dissipation de l'énergie d'un écoulement hydraulique par entrấnement d'air, j'évoquerai certains essais entrepris vers 1960 par la Direction des Etudes et Recherches d'EDF pour l'étude des "Compresseurs d'air hydrauliques à émulsion" (1). A l'entrée de ces appareils se trouve un "émulseur" chargé de réaliser une émulsion de bulles d'air (concentration volumique allant de 5 à $30 \%$ ) dans un circuit d'eau pouvant atteindre plusieurs dizaines de $\mathrm{m}^{3} / \mathrm{s}$. Malgré l'ingéniosité des nombreux types d'émulseurs testés,la perte de charge engendrée par ces appareils est toujours relativement importante et limite les applications pratiques des dits compresseurs. Les causes de cette perte d'énergie n'ont pu être clairement élucidées mais il se peut qu'elles trouvent leur origine en partie - outre les mécanismes exposés au paragraphe 3 du mémoire en discussions - dans la "fabrication" de l'émulsion de bulles d'air dans l'eau. Le travail des forces de tension superficielle (loi de Laplace) est très inférieur aux pertes d'énergie observées. L'analyse fine de celles-ci devrait conduire à des perfectionnements notables des dispositifs industriels concernés.

$M$. le Président. - Quand on examine l'influence de la présence de l'air dans les phénomènes de dissipation d'énergie, je ne puis m'empêcher d'évoquer les cas extrêmes des jets d'eau et de la chute en pluie.

M. DUPORT. - Deux problèmes viennent d'être soulevés.

M. REMENIERAS vient de parler du problème de l'introduction d'air dans l'écoulement. C'est effectivement un problème difficile. Il existe des exemples, qui ne sont pas français, de ratés de dispositifs d'aération par le radier sur des coursiers à grande vitesse. En fait, il est relativement difficile d'arriver à faire entrer l'air, même dans des zones qui sont a priori déprimées ; très souvent, on n'arrive qu'à des phénomènes instables ou à des entrées d'air en quantités très insuffisantes.

(1) Pour plus de détail, voir notamment : G. REMENIERAS. Les compresseurs d'air hydrauliques à émulsion. Chimie et Industrie Génie Chimique. Vol. 84, $\mathrm{N}^{\circ} 4$ Oct. 1960, p. 130-141. 
Je signale un problème qui n'est pas celui d'un dissipateur d'énergie : celui des chambres de mélange dans des propulseurs navals à propulsion diphasique, qui ontété très étudiés, y compris en France, il y a un peu moins de dix ans. Il faut savoir que le problème le plus difficile est cette chambre de mélange qui peut consommer beaucoup d'energie sur le circuit d'air.

M. REMENIERAS. - La fabrication d'émulsion est énergétiquement coûteuse.

M. DUPORT. - Ceci dit, si l'on commence par fragmenter l'écoulement liquide avant son émulsionnemert, on rend les choses beaucoup plus faciles.

En ce qui concerne la dissipation par l'air, j'ai moi aussi des souvenirs très anciens sur le sujet : c'est la dissipation du jet de Champ-sur-Drac, dans les années 40 . On s'était posé la question de savoir, dès ce moment-là, quel était le bilan d'énergie dans ce jet; il s'agissait de ne pas affouiller le bassin de dissipation peu profond qui était en aval.

Une première remarque avait été faite : à la formation des gouttes, la captation d'énergie par la tension superficielle est parfaitement dérisoire ; on s'était quand même donné la peine de s'en assurer. Ensuite, on a constaté que le freinage sur l'air est également tout à fait dérisoire. Cela répond à la question de M. BERGERON. En fait, le frottement sur l'air intervient malgré tout au début dans la trajectoire globale du jet, mais, dans le bilan énergétique, cela compte vraiment pour très peu. Par contre, cette projection du jet dans l'air - l'effet de dispersion, non pas de dissipation - a un effet positif puisque, au moment de la retombée dans le bassin, on a un écoulement hydraulique qui a peu dissipé son énergie, mais qui est très dissipateur après retombée dans le bassin. 